\title{
Avaliação de Diferentes Herbicidas para ERRAdicaÇão Química DE COQUEIROS INFECTADOS COM RESINOSE ${ }^{1}$
}

\author{
Herbicide Selection for Chemical Eradication of Coconut Palms Infected with Stem-Bleeding \\ FONTES, H.R. ${ }^{2}$, PROCOPIO, S.O. ${ }^{2}$, CARGNELUTTI FILHO, A. ${ }^{3}$, FERREIRA, J.M.S..$^{2}$ e \\ FERNANDES, M.F. ${ }^{2}$
}

\begin{abstract}
RESUMO - Objetivou-se neste trabalho selecionar herbicidas que possam ser utilizados na erradicação química de coqueiros infectados com resinose. O experimento foi instalado no município de Neópolis - SE, no periodo de junho a julho de 2007. Utilizou-se uma população de coqueiros da variedade anão-verde, implantada há 11 anos. O delineamento experimental foi o inteiramente casualizado, em parcelas subdivididas no tempo, com cinco repetições (cada unidade experimental foi composta por um coqueiro infectado). Os tratamentos foram formados pela combinação de dez tratamentos herbicidas \{MSMA (36 g por planta); glyphosate (18 g por planta); paraquat (10 g por planta); [2,4-D + picloram $](18+1,125 \mathrm{~g}$ por planta); $[2,4-\mathrm{D}+$ picloram $]+$ paraquat $([9+0,5625]+5 \mathrm{~g}$ por planta $) ;$ MSMA + glyphosate $(18+$ 9 g por planta); MSMA + paraquat $(18+5 \mathrm{~g}$ por planta); glyphosate + paraquat $(9+5 \mathrm{~g}$ por planta); $[2,4-\mathrm{D}+$ picloram $]+\mathrm{MSMA}([9+0,5625]+18 \mathrm{~g}$ por planta); tratamento controle (testemunha) sem aplicação\} e de quatro épocas de avaliação $(7,14,21$ e 28 dias após a aplicação dos tratamentos herbicidas). Os herbicidas foram injetados no estipe dos coqueiros em um furo de $25 \mathrm{~cm}$ de profundidade, $5 \mathrm{~cm}$ de diâmetro e inclinação de $45^{\circ}$, localizado a $1,0 \mathrm{~m}$ de altura. A aplicação foi realizada por intermédio de uma seringa graduada, imediatamente após a abertura dos furos, sendo os herbicidas aplicados sem diluição. Foram realizadas quatro avaliações da dessecação dos coqueiros, aos 7, 14, 21 e 28 dias após a aplicação dos tratamentos (DAA). O herbicida MSMA foi o que resultou na dessecação mais rápida dos coqueiros doentes, além de promover a morte de todas as plantas avaliadas, sendo por isso o tratamento mais indicado na continuação dos estudos visando à proposição de uma técnica para a erradicação química de coqueiros infectados com resinose.
\end{abstract}

Palavras-chave: Cocos nucifera, Ceratocystis paradoxa, MSMA, paraquat, glyphosate.

\begin{abstract}
The objective of this work was to select herbicides that can be used to chemically eradicate coconut palms infected with stem-bleeding. The experiment was carried out in Neopolis, Sergipe, from June to July 2007. The area had been cultivated with the coconut variety green-dwarf for 11 years. The experiment was laid out in a completely randomized design, in split-plots and five replicates (each experimental unit was composed by one coconut tree). Treatments comprised combinations of ten herbicides $\{M S M A$ (36 g per plant); glyphosate (18 g per plant); paraquat (18 g per plant); $[2,4-D+$ picloram $(18+1,125 \mathrm{~g}$ per plant); $[2,4-D+$ picloram] + paraquat $([9+0,5625]+5$ g per plant); $M S M A+$ glyphosate $(18+9$ g per plant); MSMA + paraquat $(18+$ $5 \mathrm{~g}$ per plant); glyphosate + paraquat $(9+5 \mathrm{~g}$ per plant); $[2,4-D+$ picloram $]+M S M A([9+0,5625]+$ $18 \mathrm{~g}$ per plant); control treatment with no application\} and four evaluation times (7, 14, 21 and 28 days after herbicide application). The herbicides were injected in the stem of the palm plants through a hole $25 \mathrm{~cm}$ deep $x 5 \mathrm{~cm}$ in diameter and 45 inclination, located at $1 \mathrm{~m}$ height. The application was made with a graded syringe immediately after drilling of the holes using undiluted herbicide. The herbicide MSMA resulted in the quickest desiccation of infected coconut trees and killed all the tested plants. Given these results, MSMA was chosen to be tested in further studies aiming to elaborate a technique for the chemical eradication of coconut trees infected with stem-bleeding.
\end{abstract}

Keywords: Cocos nucifera, Ceratocystis paradoxa, MSMA, paraquat, glyphosate.

1 Recebido para publicação em 7.7.2008 e na forma revisada em 13.11.2009.

2 Engo-Agr ${ }^{\circ}$, D.Sc., Pesquisador da Embrapa Tabuleiros Costeiros, 49025-040 Aracaju-SE, <procopio@cpatc.embrapa.br>; ${ }^{3}$ Eng-o-Agr ${ }^{0}$, D. Sc., Professor do Dep. de Fitotecnia, Universidade Federal de Santa Maria - UFSM, 97105-900 Santa Maria-RS. 


\section{INTRODUÇÃO}

A doença conhecida como "stem-bleeding" ou resinose, cujo agente causal é o fungo Thielaviopsis paradoxa, Chalara paradoxa ou Ceratocystis paradoxa (Dede \& Okungbowa, 2007), é relatada como uma das principais doenças do coqueiro no mundo (Ramírez Naranjo, 1991). Ao longo da década atual, a doença foi detectada em coqueirais de Sergipe e tem se disseminado rapidamente, causando prejuízos significativos à produtividade da cultura neste Estado (Warwick et al., 2004). $\mathrm{O}$ coqueiro anão-verde é o cultivar predominantemente plantado no Platô de Neópolis (Neópolis-SE), a principal região produtora desta cultura no Estado de Sergipe. Nesta região, mais de $79 \%$ dos coqueirais são formados por essa variedade, que, por se tratar de um material genético mais homogêneo, tem demonstrado maior suscetibilidade às doenças em geral (Fontes \& Wanderley, 2006). Cerca de $99 \%$ das plantas infectadas em Sergipe foram registradas em lotes cultivados com esse cultivar. No Brasil, a ocorrência dessa doença em coqueiro tem sido observada nos seguintes Estados e municipios: Sergipe (Neópolis, Aracaju, Malhador, Ilha das Flores e Canindé do São Francisco), Bahia (Una, Porto Seguro, Canavieiras, Itacaré, Camaçari, Rio Real, Anagé e Jeremoabo), Alagoas (São Sebastião) e Pernambuco (Petrolina) (Ferreira $\&$ Fontes, 2007). Além do coqueiro, a doença é também relatada em outras palmeiras no Havaí (Nelson, 2005), nas Filipinas (Lamberti et al., 1985), na Tailândia (Soytong et al., 2005) e no México (Ramírez et al., 2002), causando grandes devastações.

Não há relatos de produtos químicos capazes de curar a resinose em plantas com sinais avançados da doença ou de impedir sua disseminação na plantação. Por isso, atualmente, recomenda-se a erradicação mecânica e destruição das plantas severamente infestadas (queima), removendo o máximo possivel de raízes, a fim de se evitar a disseminação da doença, principalmente pelo inseto-vetor Rhynchophorus palmarum (Parra et al., 2003). Todavia, esse processo é extremamente trabalhoso e oneroso, demandando grande quantidade de mão de obra, além da dificuldade da queima das plantas doentes no periodo chuvoso. Uma alternativa a essa técnica de eliminação mecânica/física pode ser a erradicação química. O uso de herbicidas para controle de espécies arbóreas já vem sendo adotado em pastagens (Carmona et al., 2001), para evitar a rebrota de árvores de interesse econômico após seu corte (Loftis, 1985; Souza et al., 2006), e no controle de espécies utilizadas na produção de narcóticos (Ferreira \& Reddy, 2000).

Além da eficiência dos herbicidas em erradicar as plantas, um outro fator a ser considerado nesse tipo de operação é a velocidade de dessecação das plantas após a aplicação dos produtos, já que herbicidas de efeito mais lento proporcionariam maior tempo de multiplicação de R. palmarum e, consequentemente, maior possibilidade de disseminação do agente causal por esses insetos. A aplicação de herbicidas em coníferas já foi avaliada por Lanier (1989) como estratégia para conter a disseminação do patógeno Ceratocystis ulmi, por meio do controle das larvas do besouro vetor Scolytus multistriatus alojadas em galerias nos troncos dessas árvores.

O objetivo deste trabalho foi selecionar herbicidas que possam ser utilizados na erradicação química de coqueiros infectados com resinose, adotando-se como critérios de seleção a eficiência de erradicação e a velocidade de dessecação das plantas infectadas com resinose.

\section{MATERIAL E MÉTODOS}

O experimento foi instalado no município de Neópolis-SE $\left(10^{\circ} 19^{\prime}\right.$ de latitude sul, 36³4' de longitude oeste e $30 \mathrm{~m}$ de altitude) no periodo de junho a julho de 2007. O clima local, pela classificação de Köppen, é do tipo AS' (tropical chuvoso com verão seco) e o solo da área experimental, classificado como Argissolo Amarelo distrófico coeso (Araújo Filho et al., 1999). O coqueiral foi composto pela variedade anão-verde, tendo sido implantado há 11 anos no espaçamento de $7,5 \times 7,5 \times 7,5 \mathrm{~m}$, em triângulo.

O delineamento experimental utilizado foi o inteiramente casualizado (DIC), em parcelas subdivididas no tempo, com cinco repetições (cada unidade experimental foi composta por um coqueiro infectado). Os tratamentos foram formados pela combinação de dez 
herbicidas \{MSMA (36 g por planta); glyphosate (18 g por planta); paraquat ( $10 \mathrm{~g}$ por planta); [2,4-D + picloram] $(18+1,125 \mathrm{~g}$ por planta $)$; $[2,4-\mathrm{D}+$ picloram $]+$ paraquat $([9+0,5625]+$ $5 \mathrm{~g}$ por planta); MSMA + glyphosate $(18+9 \mathrm{~g}$ por planta); MSMA + paraquat $(18+5$ g por planta); glyphosate + paraquat $(9+5$ g por planta $)$; $[2,4-\mathrm{D}+$ picloram $]+\operatorname{MSMA}([9+0,5625]+$ $18 \mathrm{~g}$ por planta); tratamento controle (testemunha) sem aplicação\} e quatro épocas de avaliação $(7,14,21$ e 28 dias após a aplicação dos tratamentos herbicidas).

Os herbicidas foram injetados no estipe dos coqueiros em um furo de $25 \mathrm{~cm}$ de profundidade e $5 \mathrm{~cm}$ de diâmetro, localizado a 1,0 m de altura. A aplicação foi realizada por intermédio de uma seringa graduada, imediatamente após a abertura dos furos, sendo os herbicidas aplicados sem diluição. Quando o tratamento constava de mais de um herbicida, a aplicação foi realizada sequencialmente. Para não haver vazamento do herbicida aplicado, os furos foram abertos obedecendo a um ângulo de inclinação de $45^{\circ}$. Após a injeção dos herbicidas, os furos foram fechados com argila umedecida.

Foram realizadas quatro avaliações visuais da dessecação das folhas 1,9 e 14 e do cacho 14 , a contar do ápice; e uma avaliação da dessecação geral das plantas, aos 7, 14, 21 e 28 dias após a aplicação dos tratamentos (DAA), utilizando-se escala percentual, em que $100 \%$ significa morte da planta, inclusive com queda completa da copa, e 0\% (zero), ausência de sintomas do tratamento herbicida.

Os dados coletados foram submetidos à análise de variância e teste $\mathrm{F}$ a $5 \%$ de significância. A análise dos efeitos significativos dos tratamentos herbicidas dentro de cada época de avaliação foi realizada pelo teste de Scott Knott a 5\% de significância, e o comportamento dos tratamentos herbicidas ao longo do tempo foi avaliado por análise de regressão.

\section{RESULTADOS E DISCUSSÃO}

Verificou-se interação entre o comportamento dos herbicidas e as épocas de avaliação para todos os parâmetros avaliados, mostrando que a eficácia dos herbicidas variou ao longo do tempo.
Inicialmente, verificou-se que a "folha 1" apresentou os menores niveis de sintomas, mostrando possivelmente a maior dificuldade da translocação dos herbicidas até esta folha, que é a mais distante do local de aplicação dos produtos. Aos 7 dias após a aplicação dos tratamentos com herbicidas (DAA), o glyphosate + paraquat e MSMA + paraquat foram os que apresentaram maior capacidade de dessecação da "folha 1", mesmo assim em niveis bastante discretos: 36,0 e $31,0 \%$, respectivamente (Tabela 1). Esses mesmos tratamentos continuaram apresentando os maiores sintomas na "folha 1" dos coqueiros doentes na avaliação realizada aos 14 DAA. Contudo, aos 21 DAA, a aplicação isolada de MSMA e a associação de glyphosate e paraquat foram os tratamentos que se destacaram na dessecação da "folha 1" (Tabela 1 e Figura 1). MSMA foi o único tratamento a dessecar completamente a "folha 1" de todas as repetições aos 28 DAA, não diferindo, porém, dos efeitos observados dos tratamentos paraquat, glyphosate + paraquat, glyphosate e [2,4-D + picloram $]+$ paraquat (Tabela 1 e Figura 1). Verifica-se que o tratamento que apresentou o pior desempenho na dessecação da "folha 1 " dos coqueiros infectados com resinose foi [2,4-D + picloram] + MSMA, cuja dessecação final (28 DAA) não ultrapassou 24\% (Tabela 1 e Figura 1), ou seja, resultado inaceitável para qualquer pretensão de uso nessa modalidade.

Os efeitos dos tratamentos herbicidas foram muito semelhantes sobre as "folhas $9 \mathrm{e}$ 14", conforme observado nas Tabelas 2 e 3 e nas Figuras 2 e 3. O tratamento que resultou em dessecação inicial mais intensa foi a aplicação isolada do MSMA, que proporcionou dessecação média de 70,0 e 72,0\%, respectivamente para as "folhas 9 e 14", aos 7 DAA. Todavia, na avaliação subsequente (14 DAA), outros tratamentos, como glyphosate + paraquat, [2,4-D + picloram] + paraquat, MSMA + glyphosate e MSMA + paraquat, também apresentaram resultados satisfatórios, não diferindo da aplicação isolada do MSMA (Tabelas 2 e 3). Aos 21 DAA, além desses tratamentos, a aplicação isolada de glyphosate obteve destaque na dessecação dos coqueiros infectados com resinose. Esses seis tratamentos com a inclusão da aplicação isolada de paraquat se mantiveram como os mais eficientes ao término das avaliações (28 DAA), 
Tabela 1 - Dessecação da “folha 1" de coqueiros (variedade anão-verde) por herbicidas injetados no estipe. Neópolis-SE. 2007

\begin{tabular}{|c|c|c|c|c|c|c|c|}
\hline \multirow{2}{*}{ Herbicida } & \multirow{2}{*}{$\begin{array}{c}\text { Dose } \\
\text { (g por planta) }\end{array}$} & $7 \mathrm{DAA}^{1 /}$ & $14 \mathrm{DAA}$ & $21 \mathrm{DAA}$ & 28 DAA & \multirow{2}{*}{ Equação } & \multirow{2}{*}{$\mathrm{R}^{2}$} \\
\hline & & \multicolumn{4}{|c|}{ Dessecação (\%) } & & \\
\hline MSMA & 36 & $17,0 \mathrm{~b}$ & $27,0 \mathrm{c}$ & $94,0 \mathrm{a}$ & $100,0 \mathrm{a}$ & $\hat{\mathrm{Y}}=16,8744+83,1970 /\left(1+\exp \left(-\left(\mathrm{X}^{*}-17,0620\right) / 1,5493\right)\right)$ & 1,00 \\
\hline Glyphosate & 18 & $9,0 \mathrm{c}$ & $36,0 \mathrm{~b}$ & $80,0 \mathrm{~b}$ & 95,8 a & $\hat{Y}=99,8066 /\left(1+\exp \left(-\left(X^{*}-15,9969\right) / 3,6825\right)\right)$ & 0,99 \\
\hline Paraquat & 10 & $2,0 \mathrm{c}$ & $9,0 \mathrm{~d}$ & $52,0 \mathrm{~d}$ & $98,4 \mathrm{a}$ & $\hat{\mathrm{Y}}=109,5593 /\left(1+\exp \left(-\left(\mathrm{X}^{*}-21,3166\right) / 3,0689\right)\right)$ & 0,99 \\
\hline [2,4-D + picloram] & {$[18+1,125]$} & $23,0 \mathrm{~b}$ & $41,0 \mathrm{~b}$ & $49,6 \mathrm{~d}$ & $88,0 \mathrm{~b}$ & $\hat{\mathrm{Y}}=3157,6331 /\left(1+\exp \left(-\left(\mathrm{X}^{*}-84,4894\right) / 15,7885\right)\right)$ & 0,97 \\
\hline$[2,4-\mathrm{D}+$ picloram $]+$ paraquat & {$[9+0,5625]+5$} & $10,0 \mathrm{c}$ & $39,0 \mathrm{~b}$ & $69,0 \mathrm{c}$ & $94,4 \mathrm{a}$ & $\hat{\mathrm{Y}}=105,4952 /\left(1+\exp \left(-\left(\mathrm{X}^{*}-17,3175\right) / 5,1526\right)\right)$ & 0,99 \\
\hline MSMA + glyphosate & $18+9$ & $22,0 \mathrm{~b}$ & $39,0 \mathrm{~b}$ & $43,8 \mathrm{~d}$ & $89,0 \mathrm{~b}$ & $\hat{\mathrm{Y}}=3546,7314 /\left(1+\exp \left(-\left(\mathrm{X}^{*}-81,1952\right) / 14,3742\right)\right)$ & 0,94 \\
\hline MSMA + paraquat & $18+5$ & $31,0 \mathrm{a}$ & $52,0 \mathrm{a}$ & $66,4 \mathrm{c}$ & $84,6 \mathrm{~b}$ & $\hat{\mathrm{Y}}=108,3148 /\left(1+\exp \left(-\left(\mathrm{X}^{*}-15,6418\right) / 9,9886\right)\right)$ & 0,99 \\
\hline Glyphosate + paraquat & $9+5$ & $36,0 \mathrm{a}$ & $48,0 \mathrm{a}$ & $91,0 \mathrm{a}$ & $96,4 \mathrm{a}$ & $\hat{\mathrm{Y}}=35,6042+60,9520 /\left(1+\exp \left(-\left(\mathrm{X}^{*}-16,6078\right) / 1,9100\right)\right)$ & 1,00 \\
\hline$[2,4-\mathrm{D}+$ picloram $]+\mathrm{MSMA}$ & {$[9+0,5625]+18$} & $5,0 \mathrm{c}$ & $8,8 \mathrm{~d}$ & $10,0 \mathrm{e}$ & $23,8 \mathrm{c}$ & $\hat{Y}=1501,1857 /\left(1+\exp \left(-\left(X^{*}-77,5672\right) / 11,8851\right)\right)$ & 0,93 \\
\hline Testemunha & - & $0,0 \mathrm{c}$ & $0,0 \mathrm{~d}$ & $0,0 \mathrm{e}$ & $0,0 \mathrm{~d}$ & - & - \\
\hline
\end{tabular}

Médias seguidas pelas mesmas letras na coluna não diferem estatisticamente entre si pelo teste de Scott Knott a 5\% de probabilidade.

1/ DAA $=$ dias após a aplicação. Estimativa dos parâmetros das equações significativos a $5 \%$ pelo teste $\mathrm{t}$.

Tabela 2 - Dessecação da “folha 9" de coqueiros (variedade anão-verde) por herbicidas injetados no estipe. Neópolis-SE. 2007

\begin{tabular}{|c|c|c|c|c|c|c|c|}
\hline \multirow{2}{*}{ Herbicida } & \multirow{2}{*}{$\begin{array}{c}\text { Dose } \\
\text { (g por planta) }\end{array}$} & $7 \mathrm{DAA}^{\frac{1}{1}}$ & $14 \mathrm{DAA}$ & $21 \mathrm{DAA}$ & $28 \mathrm{DAA}$ & \multirow{2}{*}{ Equação } & \multirow{2}{*}{$\mathrm{R}^{2}$} \\
\hline & & \multicolumn{4}{|c|}{ Dessecação (\%) } & & \\
\hline MSMA & 36 & $70,0 \mathrm{a}$ & $85,0 \mathrm{a}$ & $94,6 \mathrm{a}$ & $100,0 \mathrm{a}$ & $\hat{\mathrm{Y}}=105,4200 /\left(1+\exp \left(-\left(\mathrm{X}^{*}-0,5910\right) / 9,4064\right)\right)$ & 0,99 \\
\hline Glyphosate & 18 & $15,0 \mathrm{e}$ & $50,0 \mathrm{~b}$ & $85,0 \mathrm{a}$ & $99,0 \mathrm{a}$ & $\hat{\mathrm{Y}}=102,4623 /\left(1+\exp \left(-\left(\mathrm{X}^{*}-14,2618\right) / 4,1800\right)\right)$ & 0,99 \\
\hline Paraquat & 10 & $2,6 \mathrm{f}$ & $10,0 \mathrm{~d}$ & $47,0 \mathrm{~b}$ & $90,6 \mathrm{a}$ & $\hat{\mathrm{Y}}=106,2959 /\left(1+\exp \left(-\left(\mathrm{X}^{*}-21,8281\right) / 3,5182\right)\right)$ & 0,99 \\
\hline$[2,4-\mathrm{D}+$ picloram $]$ & {$[18+1,125]$} & $22,0 \mathrm{~d}$ & $45,0 \mathrm{~b}$ & $55,0 \mathrm{~b}$ & $71,0 \mathrm{~b}$ & $\hat{\mathrm{Y}}=79,5061 /\left(1+\exp \left(-\left(\mathrm{X}^{*}-13,3720\right) / 7,4824\right)\right)$ & 0,97 \\
\hline$[2,4-\mathrm{D}+$ picloram $]+$ paraquat & {$[9+0,5625]+5$} & $47,0 \mathrm{c}$ & $89,0 \mathrm{a}$ & $90,8 \mathrm{a}$ & $95,2 \mathrm{a}$ & $\hat{\mathrm{Y}}=93,2320 /\left(1+\exp \left(-\left(\mathrm{X}^{*}-6,9592\right) / 2,3542\right)\right)$ & 0,99 \\
\hline MSMA + glyphosate & $18+9$ & $58,0 \mathrm{~b}$ & $83,0 \mathrm{a}$ & 87,4 a & $95,0 \mathrm{a}$ & $\hat{\mathrm{Y}}=93,8835 /\left(1+\exp \left(-\left(\mathrm{X}^{*}-4,5265\right) / 5,0077\right)\right)$ & 0,97 \\
\hline MSMA + paraquat & $18+5$ & $57,0 \mathrm{~b}$ & $79,0 \mathrm{a}$ & 86,4 a & 94,6 a & $\hat{\mathrm{Y}}=96,2785 /\left(1+\exp \left(-\left(\mathrm{X}^{*}-4,3574\right) / 6,7840\right)\right)$ & 0,98 \\
\hline Glyphosate + paraquat & $9+5$ & $39,0 \mathrm{c}$ & $90,0 \mathrm{a}$ & 92,6 a & $98,0 \mathrm{a}$ & $\hat{\mathrm{Y}}=95,5613 /\left(1+\exp \left(-\left(\mathrm{X}^{*}-7,8347\right) / 2,2530\right)\right)$ & 0,99 \\
\hline [2,4-D + picloram $]+$ MSMA & {$[9+0,5625]+18$} & $28,8 \mathrm{~d}$ & $33,8 \mathrm{c}$ & $34,2 \mathrm{c}$ & $48,8 \mathrm{c}$ & $\hat{Y}=914,3423 /\left(1+\exp \left(-\left(X^{*}-140,3648\right) / 38,3586\right)\right)$ & 0,85 \\
\hline Testemunha & - & $0,0 \mathrm{f}$ & $0,0 \mathrm{e}$ & $0,0 \mathrm{~d}$ & $0,0 \mathrm{~d}$ & - & - \\
\hline
\end{tabular}

Médias seguidas pelas mesmas letras na coluna não diferem estatisticamente entre si pelo teste de Scott Knott a 5\% de probabilidade. ${ }^{1 /} \mathrm{DAA}=$ dias após a aplicação. *Estimativa dos parâmetros das equações significativos a $5 \%$ pelo teste $\mathrm{t}$.

Tabela 3 - Dessecação da "folha 14" de coqueiros (variedade anão-verde) por herbicidas injetados no estipe. Neópolis-SE. 2007

\begin{tabular}{|c|c|c|c|c|c|c|c|}
\hline \multirow{2}{*}{ Herbicida } & \multirow{2}{*}{ Dose (g por planta) } & $7 \mathrm{DAA}^{1 /}$ & 14 DAA & $21 \mathrm{DAA}$ & $28 \mathrm{DAA}$ & \multirow{2}{*}{ Equação } & \multirow[t]{2}{*}{$\mathrm{R}^{2}$} \\
\hline & & \multicolumn{4}{|c|}{ Dessecação (\%) } & & \\
\hline MSMA & 36 & $72,0 \mathrm{a}$ & $79,0 \mathrm{a}$ & $92,0 \mathrm{a}$ & $100,0 \mathrm{a}$ & $\hat{\mathrm{Y}}=208,7336 /\left(1+\exp \left(-\left(\mathrm{X}^{*}-30,7064\right) / 36,1981\right)\right)$ & 0,98 \\
\hline Glyphosate & 18 & $15,0 \mathrm{e}$ & $56,0 \mathrm{~b}$ & $84,0 \mathrm{a}$ & 99,0 a & $\hat{\mathrm{Y}}=99,6799 /\left(1+\exp \left(-\left(\mathrm{X}^{*}-13,3578\right) / 3,9752\right)\right)$ & 0,99 \\
\hline Paraquat & 10 & $1,0 \mathrm{f}$ & $11,0 \mathrm{~d}$ & $37,0 \mathrm{c}$ & $93,0 \mathrm{a}$ & $\hat{\mathrm{Y}}=116,5069 /\left(1+\exp \left(-\left(\mathrm{X}^{*}-22,7565\right) / 3,8145\right)\right)$ & 0,99 \\
\hline [2,4-D + picloram] & {$[18+1,125]$} & $24,0 \mathrm{~d}$ & $40,0 \mathrm{c}$ & $54,0 \mathrm{~d}$ & $73,0 \mathrm{~b}$ & $\hat{\mathrm{Y}}=120,2114 /\left(1+\exp \left(-\left(\mathrm{X}^{*}-22,9615\right) / 11,8571\right)\right)$ & 0,99 \\
\hline$[2,4-\mathrm{D}+$ picloram $]+$ paraquat & {$[9+0,5625]+5$} & $40,0 \mathrm{c}$ & $81,0 \mathrm{a}$ & $88,0 \mathrm{a}$ & $95,0 \mathrm{a}$ & $\hat{\mathrm{Y}}=92,8071 /\left(1+\exp \left(-\left(\mathrm{X}^{*}-7,8911\right) / 3,3047\right)\right)$ & 0,99 \\
\hline MSMA + glyphosate & $18+9$ & $58,0 \mathrm{~b}$ & $87,0 \mathrm{a}$ & 90,4 a & $97,0 \mathrm{a}$ & $\hat{\mathrm{Y}}=95,0553 /\left(1+\exp \left(-\left(\mathrm{X}^{*}-5,2300\right) / 3,8887\right)\right)$ & 0,98 \\
\hline MSMA + paraquat & $18+5$ & $58,0 \mathrm{~b}$ & $78,0 \mathrm{a}$ & $83,8 \mathrm{a}$ & $95,0 \mathrm{a}$ & $\hat{\mathrm{Y}}=100,1838 /\left(1+\exp \left(-\left(\mathrm{X}^{*}-3,8014\right) / 9,1258\right)\right)$ & 0,97 \\
\hline Glyphosate + paraquat & $9+5$ & $32,0 \mathrm{~d}$ & $88,0 \mathrm{a}$ & $93,6 \mathrm{a}$ & $99,2 \mathrm{a}$ & $\hat{\mathrm{Y}}=96,8089 /\left(1+\exp \left(-\left(\mathrm{X}^{*}-8,6589\right) / 2,3598\right)\right)$ & 0,99 \\
\hline$[2,4-\mathrm{D}+$ picloram $]+$ MSMA & {$[9+0,5625]+18$} & $30,0 \mathrm{~d}$ & $38,8 \mathrm{c}$ & $41,2 \mathrm{c}$ & $53,2 \mathrm{c}$ & $\hat{\mathrm{Y}}=328,1722 /\left(1+\exp \left(-\left(\mathrm{X}^{*}-85,0603\right) / 34,2201\right)\right)$ & 0,95 \\
\hline Testemunha & & $0,0 \mathrm{f}$ & $0,0 \mathrm{e}$ & $0,0 \mathrm{~d}$ & $0,0 \mathrm{~d}$ & - & - \\
\hline
\end{tabular}

Médias seguidas pelas mesmas letras na coluna não diferem estatisticamente entre si pelo teste de Scott Knott a 5\% de probabilidade

${ }^{1 /} \mathrm{DAA}=$ dias após a aplicação. ${ }^{*}$ Estimativa dos parâmetros das equações significativos a $5 \%$ pelo teste t. 

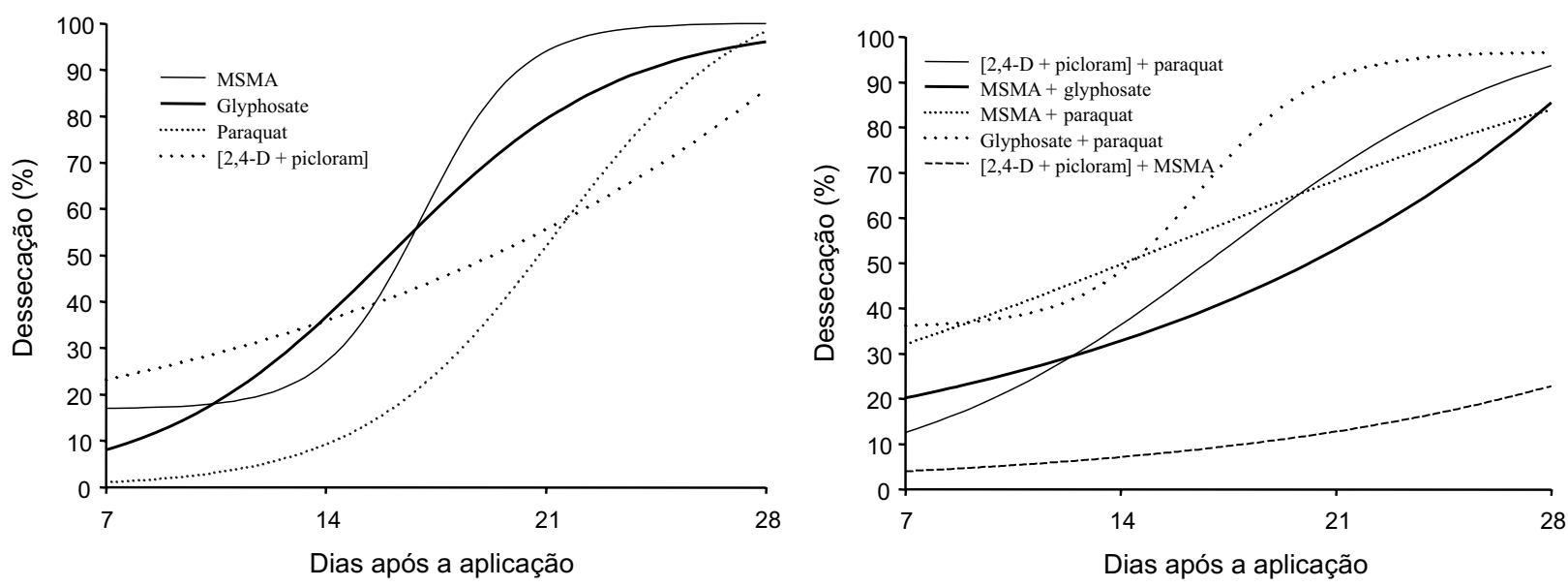

Figura 1 - Dessecação da "folha 1" de coqueiros (variedade anão-verde) após aplicação de diferentes tratamentos herbicidas, injetados no estipe das plantas. Neópolis-SE. 2007.
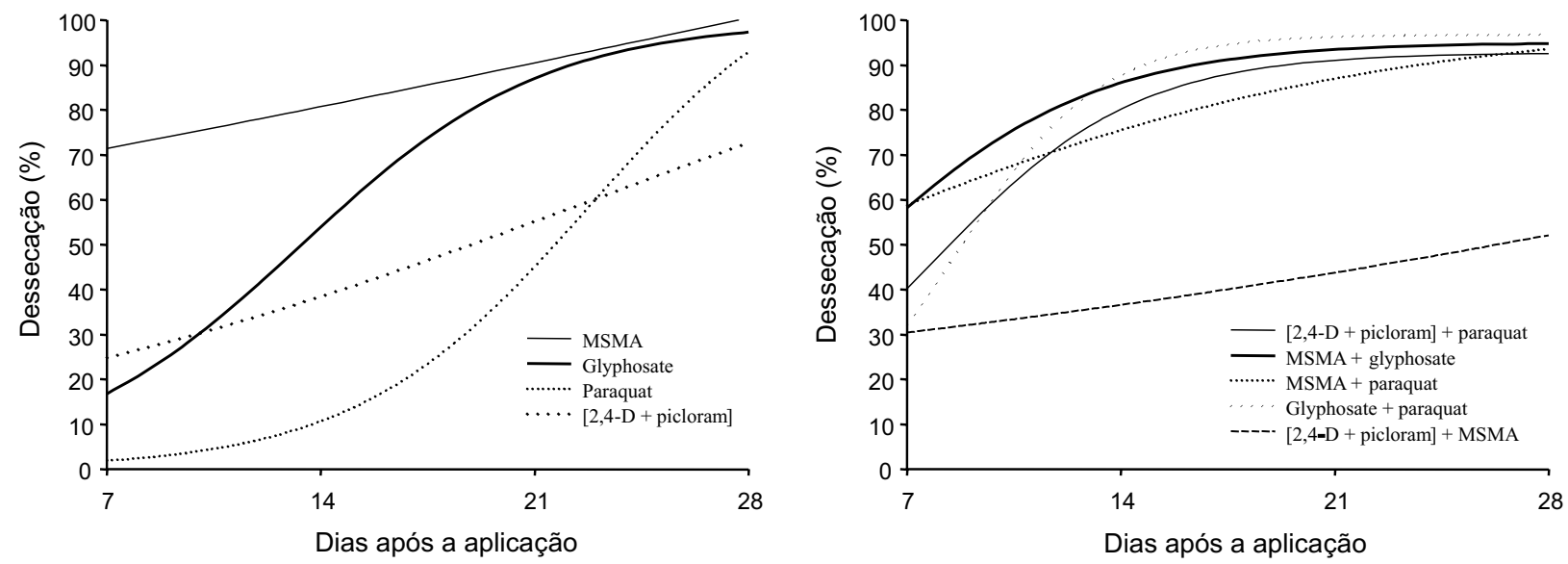

Figura 2 - Dessecação da "folha 9" de coqueiros (variedade anão-verde) após aplicação de diferentes tratamentos herbicidas, injetados no estipe das plantas. Neópolis-SE. 2007
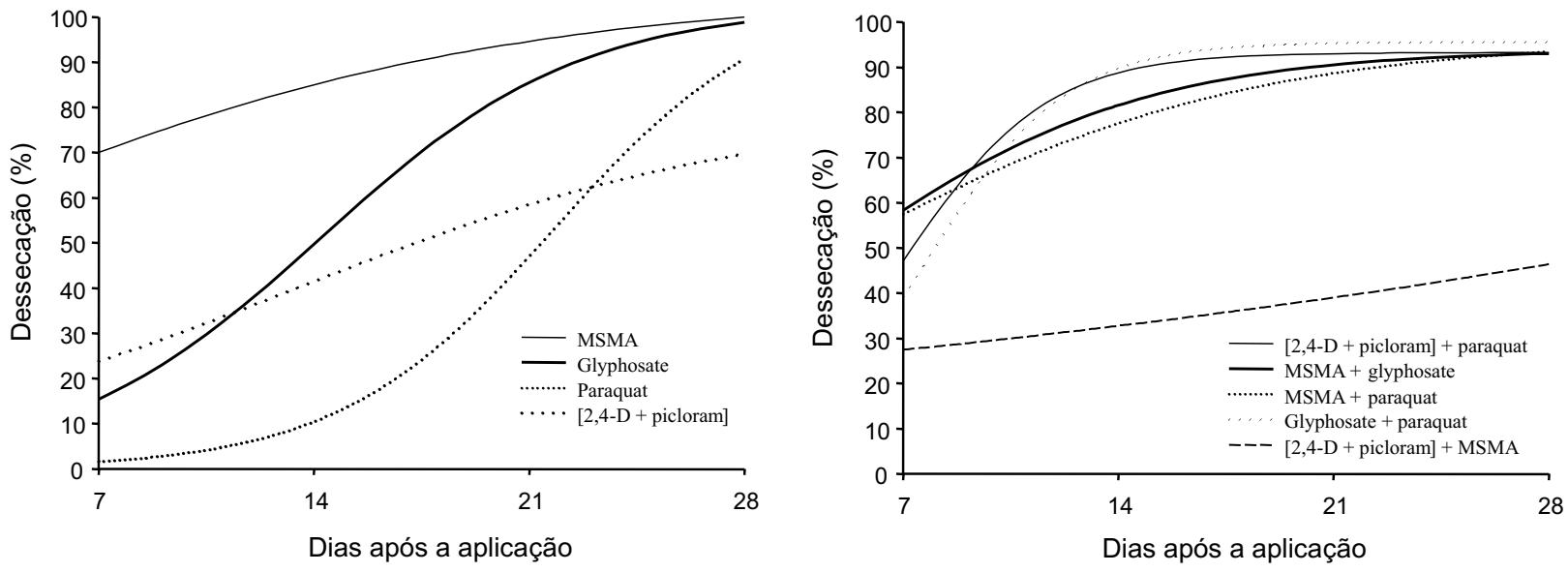

Figura 3 - Dessecação "da folha 14" de coqueiros (variedade anão-verde) após aplicação de diferentes tratamentos herbicidas, injetados no estipe das plantas. Neópolis-SE. 2007. 
apresentando dessecação média acima de $90 \%$; contudo, novamente, apenas o tratamento MSMA dessecou completamente as "folhas 9 e 14" dos coqueiros doentes. Também, foi constatada na avaliação das "folhas 9 e 14" a baixa eficiência da mistura entre os herbicidas [2,4-D + picloram] e MSMA, corroborando o observado para a "folha 1" (Tabelas 2 e 3 e Figuras 2 e 3). Esse resultado mostra uma interação antagonística entre esses dois herbicidas, pois verifica-se que o resultado final da dessecação é muito inferior ao resultado observado quando esses compostos foram aplicados isoladamente. Herbicidas que causam destruição da membrana celular, como o MSMA e o paraquat, frequentemente reduzem a absorção e a translocação de outros herbicidas aplicados em mistura (Olson \& Nalewaja, 1982; Pereira \& Crabtree, 1986; Croon et al., 1989; Culpepper et al., 1999; Burke et al., 2007), fato que pode ter sido a causa do efeito antagônico observado, porém isso não ocorreu nas demais associações envolvendo os herbicidas MSMA e paraquat.

A aplicação isolada de glyphosate foi o tratamento que promoveu maior intensidade de sintomas ao "cacho 14" dos coqueiros infectados com resinose, aos 7 DAA (Tabela 4 e Figura 4). Esse resultado pode ser explicado pela alta mobilidade do glyphosate junto ao fluxo de fotoassimilados (floema), o que resulta em

Tabela 4 - Dessecação do "cacho 14" de coqueiros (variedade anão-verde) por herbicidas injetados no estipe. Neópolis-SE. 2007

\begin{tabular}{|c|c|c|c|c|c|c|c|}
\hline \multirow{2}{*}{ Herbicida } & \multirow{2}{*}{ Dose (g por planta) } & $7 \mathrm{DAA}^{1 /}$ & $14 \mathrm{DAA}$ & $21 \mathrm{DAA}$ & $28 \mathrm{DAA}$ & \multirow{2}{*}{ Equação } & \multirow{2}{*}{$\mathrm{R}^{2}$} \\
\hline & & \multicolumn{4}{|c|}{ Dessecação (\%) } & & \\
\hline MSMA & 36 & $0,0 \mathrm{~b}$ & $58,0 \mathrm{~b}$ & $90,0 \mathrm{a}$ & $100,0 \mathrm{a}$ & $\hat{\mathrm{Y}}=95,5530 /\left(1+\exp \left(-\left(\mathrm{X}^{*}-13,3105\right) / 1,6444\right)\right)$ & 0,99 \\
\hline Glyphosate & 18 & $31,0 \mathrm{a}$ & $74,0 \mathrm{a}$ & $88,0 \mathrm{a}$ & $100,0 \mathrm{a}$ & $\hat{\mathrm{Y}}=96,8164 /\left(1+\exp \left(-\left(\mathrm{X}^{*}-10,3877\right) / 3,3428\right)\right)$ & 0,98 \\
\hline Paraquat & 10 & $0,0 \mathrm{~b}$ & $9,0 \mathrm{~d}$ & $66,0 \mathrm{c}$ & $91,0 \mathrm{a}$ & $\hat{\mathrm{Y}}=92,5729 /\left(1+\exp \left(-\left(\mathrm{X}^{*}-18,9735\right) / 2,2262\right)\right)$ & 0,99 \\
\hline$[2,4-\mathrm{D}+$ picloram $]$ & {$[18+1,125]$} & $0,0 \mathrm{~b}$ & $37,0 \mathrm{c}$ & $78,0 \mathrm{~b}$ & 83,0 a & $\hat{\mathrm{Y}}=81,9510 /\left(1+\exp \left(-\left(\mathrm{X}^{*}-14,4088\right) / 1,9471\right)\right)$ & 0,99 \\
\hline$[2,4-\mathrm{D}+$ picloram $]+$ paraquat & {$[9+0,5625]+5$} & $0,0 \mathrm{~b}$ & $44,0 \mathrm{c}$ & $84,0 \mathrm{a}$ & $93,0 \mathrm{a}$ & $\hat{\mathrm{Y}}=90,7121 /\left(1+\exp \left(-\left(\mathrm{X}^{*}-14,2145\right) / 2,1757\right)\right)$ & 0,99 \\
\hline MSMA + glyphosate & $18+9$ & $2,0 \mathrm{~b}$ & $74,0 \mathrm{a}$ & $84,0 \mathrm{a}$ & $96,0 \mathrm{a}$ & $\hat{\mathrm{Y}}=90,0987 /\left(1+\exp \left(-\left(\mathrm{X}^{*}-11,9412\right) / 1,3566\right)\right)$ & 0,98 \\
\hline MSMA + paraquat & $18+5$ & $0,0 \mathrm{~b}$ & $74,0 \mathrm{a}$ & $89,0 \mathrm{a}$ & 95,6 a & $\hat{\mathrm{Y}}=92,3091 /\left(1+\exp \left(-\left(\mathrm{X}^{*}-12,6948\right) / 0,9349\right)\right)$ & 0,99 \\
\hline Glyphosate + paraquat & $9+5$ & $8,0 \mathrm{~b}$ & $72,0 \mathrm{a}$ & $89,4 \mathrm{a}$ & 97,6 a & $\hat{\mathrm{Y}}=94,1091 /\left(1+\exp \left(-\left(\mathrm{X}^{*}-11,6691\right) / 2,0122\right)\right)$ & 0,99 \\
\hline [2,4-D + picloram] + MSMA & {$[9+0,5625]+18$} & $0,0 \mathrm{~b}$ & $5,0 \mathrm{~d}$ & $10,0 \mathrm{~d}$ & $45,0 \mathrm{~b}$ & $\hat{\mathrm{Y}}=773,1621 /\left(1+\exp \left(-\left(\mathrm{X}^{*}-41,4815\right) / 4,8300\right)\right)$ & 0,99 \\
\hline Testemunha & - & $0,0 \mathrm{~b}$ & $0,0 \mathrm{~d}$ & $0,0 \mathrm{~d}$ & $0,0 \mathrm{c}$ & - & - \\
\hline
\end{tabular}

Médias seguidas pelas mesmas letras na coluna não diferem estatisticamente entre si pelo teste de Scott Knott a 5\% de probabilidade.

${ }^{1 /} \mathrm{DAA}=$ dias após a aplicação. *Estimativa dos parâmetros das equações significativos a $5 \%$ pelo teste $\mathrm{t}$.

Tabela 5 -Aspecto geral da dessecação de coqueiros (variedade anão-verde) tratados com herbicidas injetados no estipe. Neópolis-SE. 2007

\begin{tabular}{|c|c|c|c|c|c|c|c|}
\hline \multirow{2}{*}{ Herbicida } & \multirow{2}{*}{ Dose (g por planta) } & $7 \mathrm{DAA}^{1 /}$ & $14 \mathrm{DAA}$ & $21 \mathrm{DAA}$ & $28 \mathrm{DAA}$ & \multirow{2}{*}{ Equação } & \multirow{2}{*}{$\mathrm{R}^{2}$} \\
\hline & & \multicolumn{4}{|c|}{ Dessecação (\%) } & & \\
\hline MSMA & 36 & $71,0 \mathrm{a}$ & $78,4 \mathrm{a}$ & $91,6 \mathrm{a}$ & $100,0 \mathrm{a}$ & $\hat{\mathrm{Y}}=204,4383 /\left(1+\exp \left(-\left(\mathrm{X}^{*}-29,1924\right) / 34,4475\right)\right)$ & 0,98 \\
\hline Glyphosate & 18 & $25,0 \mathrm{e}$ & $57,0 \mathrm{c}$ & 85,4 a & $94,0 \mathrm{a}$ & $\hat{\mathrm{Y}}=98,1449 /\left(1+\exp \left(-\left(\mathrm{X}^{*}-12,2777\right) / 4,8087\right)\right)$ & 0,99 \\
\hline Paraquat & 10 & $3,0 \mathrm{f}$ & $16,0 \mathrm{e}$ & $42,0 \mathrm{~d}$ & $87,0 \mathrm{a}$ & $\hat{\mathrm{Y}}=146,7210 /\left(1+\exp \left(-\left(\mathrm{X}^{*}-25,9461\right) / 5,4802\right)\right)$ & 0,99 \\
\hline$[2,4-\mathrm{D}+$ picloram $]$ & {$[18+1,125]$} & $40,0 \mathrm{~d}$ & $50,0 \mathrm{c}$ & $57,4 \mathrm{c}$ & $77,0 \mathrm{~b}$ & $\hat{\mathrm{Y}}=2168,0627 /\left(1+\exp \left(-\left(\mathrm{X}^{*}-132,0047\right) / 31,2898\right)\right)$ & 0,97 \\
\hline$[2,4-\mathrm{D}+$ picloram $]+$ paraquat & {$[9+0,5625]+5$} & $50,0 \mathrm{c}$ & $75,0 \mathrm{~b}$ & 84,6 a & $90,2 \mathrm{a}$ & $\hat{\mathrm{Y}}=91,0283 /\left(1+\exp \left(-\left(\mathrm{X}^{*}-5,8889\right) / 5,4185\right)\right)$ & 0,99 \\
\hline MSMA + glyphosate & $18+9$ & $57,0 \mathrm{~b}$ & $69,0 \mathrm{~b}$ & $79,0 \mathrm{~b}$ & $94,0 \mathrm{a}$ & $\hat{\mathrm{Y}}=450,7691 /\left(1+\exp \left(-\left(\mathrm{X}^{*}-75,8991\right) / 35,7853\right)\right)$ & 0,99 \\
\hline MSMA + paraquat & $18+5$ & $63,0 \mathrm{~b}$ & $75,2 \mathrm{~b}$ & $78,4 \mathrm{~b}$ & $91,2 \mathrm{a}$ & $\hat{\mathrm{Y}}=236,6513 /\left(1+\exp \left(-\left(\mathrm{X}^{*}-47,8730\right) / 41,2367\right)\right)$ & 0,95 \\
\hline Glyphosate + paraquat & $9+5$ & $39,0 \mathrm{~d}$ & $82,0 \mathrm{a}$ & $90,8 \mathrm{a}$ & $95,0 \mathrm{a}$ & $\hat{\mathrm{Y}}=93,8841 /\left(1+\exp \left(-\left(\mathrm{X}^{*}-8,0593\right) / 3,1343\right)\right)$ & 0,99 \\
\hline$[2,4-\mathrm{D}+$ picloram $]+$ MSMA & {$[9+0,5625]+18$} & $36,2 \mathrm{~d}$ & $36,6 \mathrm{~d}$ & $40,0 \mathrm{~d}$ & $42,2 \mathrm{c}$ & $\hat{Y}=183,3139 /\left(1+\exp \left(-\left(X^{*}-148,4050\right) / 99,2238\right)\right)$ & 0,93 \\
\hline Testemunha & - & $0,0 \mathrm{f}$ & $0,0 \mathrm{f}$ & $0,0 \mathrm{e}$ & $0,0 \mathrm{~d}$ & - & - \\
\hline
\end{tabular}

Médias seguidas pelas mesmas letras na coluna não diferem estatisticamente entre si pelo teste de Scott Knott a 5\% de probabilidade.

$\underline{1}^{\prime} \mathrm{DAA}=$ dias após a aplicação. *Estimativa dos parâmetros das equações significativos a $5 \%$ pelo teste $\mathrm{t}$ 
rápido acúmulo em drenos importantes, como os cachos (infrutescência). Aos 14 DAA, três das cinco associações de herbicidas avaliadas (MSMA + glyphosate, MSMA + paraquat e [2,4-D + picloram] + MSMA) se igualaram, estatisticamente, ao efeito apresentado pela aplicação isolada de glyphosate (Tabela 4 e Figura 4). Apesar de ter apresentado dessecação mais lenta nas partes reprodutivas do coqueiro, o tratamento MSMA, aos 21 DAA, acarretou dessecação média de 90,0\% do "cacho 14", sendo tal resultado semelhante ao observado na mesma data para os tratamentos glyphosate + paraquat, MSMA + paraquat, glyphosate e [2,4-D + picloram $]+$ paraquat.
Sete dias mais tarde (28 DAA), momento da finalização das avaliações, todos os tratamentos avaliados mostraram eficiência na dessecação do "cacho 14", com exceção da aplicação conjunta dos herbicidas [2,4-D + picloram] e MSMA, que resultou em dessecação menor que 50\% (Tabela 4 e Figura 4). Contudo, destaca-se que apenas os tratamentos MSMA e glyphosate apresentaram dessecação total do "cacho 14", ocasionando inclusive a queda do referido cacho em todas as repetições. $\mathrm{O}$ resultado obtido com a aplicação isolada do herbicida paraquat pode ser de certa forma surpreendente, pois esse herbicida em aplicações de pós-emergência não apresenta
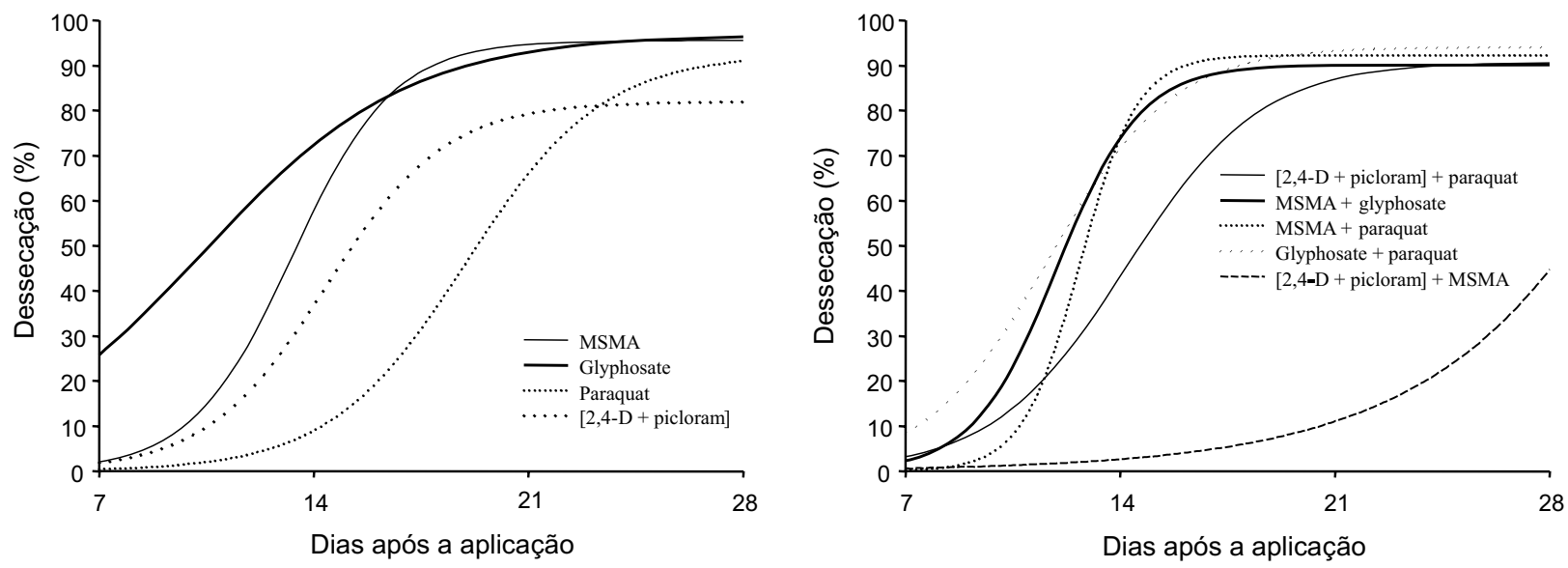

Figura 4 - Dessecação do "cacho 14" de coqueiros (variedade anão-verde) após aplicação de diferentes tratamentos herbicidas, injetados no estipe das plantas. Neópolis-SE. 2007.
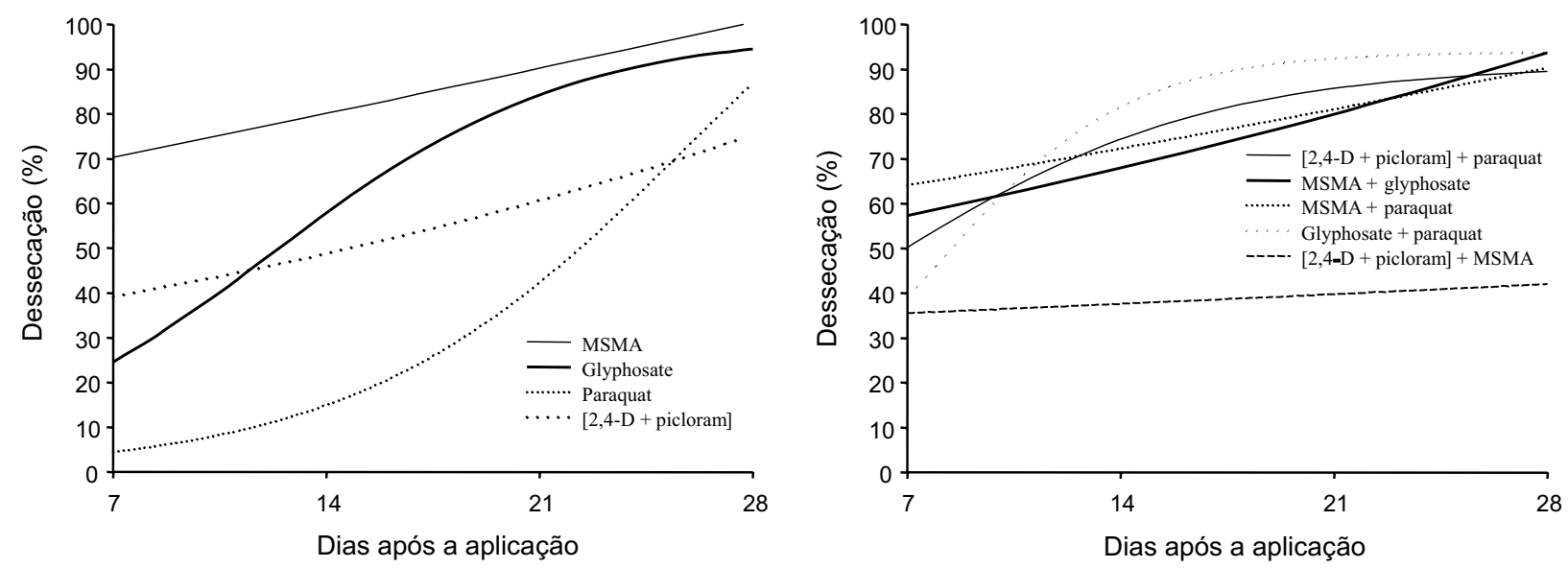

Figura 5 -Aspecto geral da dessecação de coqueiros (variedade anão-verde) tratados com herbicidas injetados no estipe das plantas Neópolis-SE. 2007. 
translocação a longa distância (Procópio et al., 2006), apenas atuando em regiões próximas ao local de interceptação das gotas oriundas da pulverização, sendo considerado um herbicida de "contato" (Gomar et al., 2004). No entanto, a provável razão dessa baixa mobilidade é o fato de o herbicida paraquat ter dificuldade de adentrar os vasos condutores, principalmente na presença de luz (Slade \& Bell, 1966). No caso da injeção no estipe do coqueiro, o herbicida já é aplicado diretamente aos vasos condutores, fazendo com que ele se redistribua a longas distâncias, chegando a promover sintomas em locais distantes de até quatro metros do local da aplicação.

Aos 7 DAA, observou-se que o tratamento à base de MSMA já resultou em média em $71,0 \%$ de dessecação das plantas de coqueiro (aspecto geral), sendo o herbicida com a ação mais rápida entre os tratamentos avaliados (Tabela 5 e Figura 5). Esse resultado pode ser considerado de extrema importância, pois para conter a disseminação da doença é essencial não somente provocar a morte das plantas doentes, mas que esse efeito dessecante seja exteriorizado o mais precocemente possivel para reduzir a atratividade da planta ao principal inseto-vetor da doença (Rhynchophorus palmarum), mesmo antes da sua erradicação total. Também, o tratamento contendo o MSMA foi o único a promover a dessecação total do coqueiro (28 DAA), inclusive com a queda de toda a copa das plantas infectadas; contudo, essa dessecação final não diferiu estatisticamente dos tratamentos glyphosate, paraquat, [2,4-D + picloram] + paraquat, MSMA + paraquat e glyphosate + paraquat (Tabela 5). Também, salienta-se que, apesar de o MSMA ter sido o tratamento mais eficiente quando aplicado isoladamente, a sua associação com o [2,4-D + picloram] promoveu queda na eficiência da dessecação (Figura 5).

Conclui-se que o MSMA é o herbicida mais apropriado para uso na erradicação química de coqueiros infectados com resinose, apesar de os tratamentos glyphosate, paraquat, [2,4-D + picloram] + paraquat, MSMA + paraquat e glyphosate + paraquat também apresentarem potencial para esse fim. Estudos posteriores para aperfeiçoar a dose e as formas de aplicação do MSMA são necessários.

\section{LITERATURA CITADA}

ARAUJO FILHO, J. C. et al. Levantamento de reconhecimento de média intensidade dos solos da região dos tabuleiros costeiros e da baixada litorânea do Estado de Sergipe. Rio de Janeiro: Embrapa Solos, 1999. (Boletim de Pesquisa). CD-ROM.

BURKE, I. C. et al. Reduced translocation is the cause of antagonism of glyphosate by MSMA in browntop millet (Brachiaria ramosa) and palmer amaranth (Amaranthus palmerii). Weed Technol., v. 21, n. 1, p. 166-170, 2007.

CARMONA, R.; ARAUJO NETO, B. S. C.; PEREIRA, R. C. Controle de Acacia farnesiana e de Mimosa pteridofita em pastagem. Pesq. Agropec. Bras., v. 36, n. 10, p. 1301-1307, 2001.

CROON, K. A.; KETCHERSID, M. L.; MERKLE, M. G. Effect of bentazon, imazaquin, and chlorimuron on the absorption and translocation of the methyl-ester of haloxyfop. Weed Sci., v. 37, n. 5, p. 645-650, 1989.

CULPEPPER, A. S. et al. Basis for antagonism in mixtures of bromoxynil plus quizalofop-P applied to yellow foxtail (Setaria glauca). Weed Technol., v. 13, n. 6, p. 515-519, 1999.

DEDE, A. P. O.; OKUNGBOWA, F. I. In vitro growth of Ceratocystis paradoxa in oil palm (Elaeis guinensis) fruit extract media. Micol. Aplicada Intern., v. 19, n. 1, p. 51-55, 2007.

FERREIRA, J. F. S.; REDDY, K. N. Absorption and translocation of glyphosate in Erythroxylum coca and E. novogranatense. Weed Sci., v. 48, n. 2, p. 193-199, 2000 .

FERREIRA, J. M. S.; FONTES, H. R. Relatório sobre o atual estágio da doença resinose no Distrito de Irrigação Platô de Neópolis. Aracaju: Embrapa Tabuleiros Costeiros, 2007. $22 \mathrm{p}$

FONTES, H. R.; WANDERLEY, M. Situação atual e perspectivas para a cultura do coqueiro no Brasil. Aracaju Embrapa Tabuleiros Costeiros, 2006. 16 p. (Embrapa

Tabuleiros Costeiros. Documentos, 94).

GOMAR, E. P. et al. Semeadura direta de forrageiras de estação fria em campo natural submetido à aplicação de herbicidas: II. Composição botânica. Ci. Rural, v. 34, n. 3, p. 769-777, 2004.

LAMBERTI, F. et al. A contribution to the etiology of the Socorro Coconut wilt disease in the Philippines.

Phytopathol. Mediterranea, v. 24, n. 3, p. 260-261, 1985.

LANIER, G. N. Trap trees for control of dutch elm disease. J. Arboric., v. 15, n. 5, p. 105-111, 1989. 
LOFTIS, D. L. Preharvest herbicide treatment improves regeneration in Southern Appalachian Hardwoods. Southern J. Appl. For., v. 9, n. 3, p. 177-180, 1985.

NELSON, S. Stem bleeding of coconut palm. Plant Dis. 2005. Disponível em: <http://www.ctahr.hawaii.edu/oc/ freepubs/pdf/PD-30.pdf>. Acesso em: 11 Dec. 2007.

OLSON, W.; NALEWAJA, J. D. Effect of MCPA on ${ }^{14}$ C-diclofop uptake translocation. Weed Sci., v. 30, n. 1, p. $59-63,1982$

PARRA, D. et al. Presencia de Thielaviopsis paradoxa De Seynes Höhn en el tubo digestivo de Rhynchophorus palmarum Linneo (Coleoptera:Curculionidae).

Entomotropica, v. 18, n. 1, p. 49-55, 2003.

PEREIRA, W.; CRABTREE, G. Absorption, translocation, and toxicity of glyphosate and oxyfluorfen in yellow nutsedge (Cyperus esculentus). Weed Sci., v. 34, n. 6, p. 923-929, 1986.

PROCÓPIO, S. O. et al. Efeitos de dessecantes no controle de plantas daninhas na cultura da soja. Planta Daninha, v. 24, n. 1, p. 193-197, 2006.
RAMÍREZ, P. A. M. et al. Incidencia de enfermedades letales en progenitores e híbridos de cocotero en Tabasco, México. R. Fitotec. Mexicana, v. 25, n. 3, p. 327-332, 2002.

RAMÍREZ NARANJO, B. N. Principales enfermedades del cocotero. ICA-Informa, v. 25, p. 5-13, 1991.

SLADE, P.; BELL, E. G. The movement of paraquat in plants. Weed Res., v. 6, n. 3, p. 267-274, 1966.

SOUZA, G. V. R. et al. Exsudato radicular de imazapyr aplicado sobre mudas de diferentes clones de eucalipto. Planta Daninha, v. 24, n. 1, p. 141-147, 2006.

SOYTONG, K.; PONGAK, W.; KASIOLARN, H. Biological control of Thielaviopsis bud rot of Hyophorbe lagenicaulis in the field. J. Agric. Technol., v. 1, n. 2, p. 235-245, 2005.

WARWICK, D. R. N.; FERREIRA, J. M. S.; PASSOS, E. E $\mathrm{M}$. Ocorrência de resinose do estipe do coqueiro em Sergipe provocada por Chalara paradoxa. Fitopatol. Bras., v. 29, Suplemento, p. 171, 2004. 